Submitted to Proc. Intl. Workshop "Lattice '89", Isola di Capri, 9/18-21/89.

\title{
LATTICE QUARK PROPAGATOR IN FIXED GAUGES"
}

\section{Claude BERNARD ${ }^{\dagger}$}

Institute of Theoretical Physics, UCSB. Santa Barbara. CA 93106

\author{
BNL- -43784
}

DE90 006767

Daniel MURPHY

Department of Physics. University of Arizona. Tucson. AX 85721

Amarjit SONI*

Department of Physics. Brookhaven National Laboratory. Upton. NY 11973

Ken YEE

Department of Physics. UCLA. Los Angeles. CA 90024

A lattice study of the quenched QCD quark propagator in Landau and Coulomb gauges is reported. We find that $m_{D}^{\text {critical }}$. the value of the quark propagator pole in the chiral limit, is $290 \pm 20 \mathrm{MeV}(\beta=5.7)$ and $350 \pm 40 \mathrm{MeV}(\beta=6.0)$. Scaling and gauge dependence of this quantity are discussed.

\section{MOTIVATION}

Although the quark propagator is not directly observable in experiment. it is a basic entity in the underlying theory. As such it has been the focus of many studies. The primary motivation for such investigations is to gain a better understanding of chiral symmetry breaking and the mechanism(s) by which it occurs. We report here on a computation of the quark propagator using Monte Carlo techniques. Clearly lattice studies can hope to give useful information to continuum models.

As a rule. most MC calculations have focused on gauge invariant quantities where no gauge fixing is necessary. By contrast. an essential feature of our quark propagator calculation is gauge fixing. Lattice gauge fixing $\mathrm{fi}_{\mathrm{c}}$ - m als: : 'lating correlation functions was pioneered by Mandula and Ogilvie who computed the gluon propagator. ${ }^{1}$ We began

\footnotetext{
-Presented by $\mathrm{A}$. Soni

tPermanent address: UCLA. Los Angeles. CA

${ }^{\star \star}$ Research supported by U.S. Department of
}

thinking about lattice gauge fixing (independently) some years ago as a technique for subtracting off $s \leftrightarrow d$ operator mixing in a lattice calculation of the $K \rightarrow \pi \pi$ decay rate. (Our experience with the current project will determine whether or not we will pursue this idea.)

More often than not the gauge of choice for theoretical investigations of chiral symmetry breaking (CSB) and dynamical mass generation is the Landau gauge, and the Dyson-Schwinger equations are a useful tool. Typically one assumes that CS is spontaneously broken (i.e., the self energy $\left.\Sigma\left(p^{2}\right) \neq 0\right) .^{2}$ The nonlinear equations allow generic solutions of two types: ${ }^{3}$

$$
\Sigma\left(p^{2}\right) \stackrel{p^{2} / \mu^{2} \gg 1}{\longrightarrow} \frac{\tilde{m}}{p^{2}}\left(p^{2} / \mu^{2}\right)^{-\epsilon} \text { or } M\left(p^{2} / \mu^{2}\right)^{-\epsilon}
$$

The first one falls rapidly with momentum as $1 / p^{2}$ and is called the regular solution. It is claimed that QCD belongs to this class of theories. Indeed experimental measurements of the electromagnetic

Energy under Contract DE-AC-02-76CH00016.

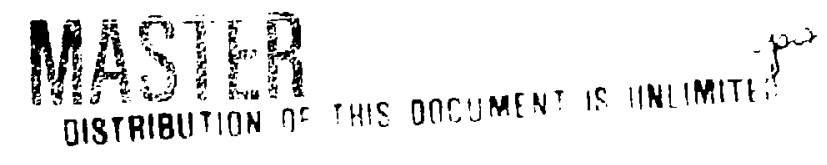


form factor of the pion are cited as being consistent with this expectation. ${ }^{4}$ It would, of course. be extremely interesting to see if direct simulation of the theory would show $\Sigma(0) \neq 0$ and to extract the momentum dependence of $\Sigma\left(p^{2}\right)$.

\section{GAUGE FIXING}

Let us cali a generic (i.e.. generated without gauge fixing) lattice gauge configuration a $\Sigma$ gauge configuration since it contains a superposition of many gauges. We have generalized the Landau gauge fixing procedure described in Mandula and Ogilvie $^{1}$ to put $\Sigma$ gauge configurations into what we call " $\lambda$ " gauges. A $\lambda$ gauge condition corresponds to the continuum requirement

$$
\lambda \partial_{t} A_{t}+\nabla \cdot \vec{A}=0 .
$$

If boundary conditions are spatially periodic and if $\lambda \neq 0$. (2) implies that

$$
\frac{\partial}{\partial t} \int_{\text {timeslice }} d^{3} \vec{x} A_{\mathrm{r}}(x)=0 .
$$

The purpose of varying $\lambda$ is to probe for possible gauge dependence of quark mass. We are studying a range of $\lambda$ 's. but only Landau and Coulomb gauge results are reported here. $\lambda=1$ corresponds to continuum Landau gauge and $\lambda=0 \lambda \rightarrow 0$ to continuum Coulomb gauge.

In Coulomb gauge fixing. there's a subtlety to the $\lambda \rightarrow 0$ limit. The $\lambda \rightarrow 0$ limit differs from the point $\lambda=0$ because condition (3) is not automatically satisfied if $\lambda=0$. Condition (3) is necessary to fix the $\vec{x}$-independent local gauge symmetry. Consequently. our Coulomb gauge fixing involves one more step than Landau gauge fixing: after fixing to $\lambda=0$. (3) is implemented as an additional subsidiary condition. Satisfying (3) requires making spatially independent gauge transformations which do not disrupt $\lambda=0$ gauge.

On the lattice.

$$
A_{\mu}(x) \equiv\left(\frac{U_{x, \mu}-U_{x, \mu}^{\dagger}}{2 i}\right)-\frac{1}{3} \text { (trace) } .
$$

The lattice realization of $(2)$ is achieved by maximizing

$Z[V]=\sum_{x}\left\{\lambda\left[V_{x} U_{x, \hat{i}} V_{x+\bar{t}}^{\dagger}\right]+\sum_{i=1}^{3}\left[V_{x} U_{x, i} V_{x+\bar{i}}^{\dagger}\right]\right\}+$ h.c. where $V_{x}$ is a gauge transformation at site $x . Z[V]$ is maximized site by site using an algorithm which sweeps repeatedly through the lattice until $V$ stablizes. A convenient measure of how well our gauge configurations satisfy (2) is

$$
\epsilon_{\lambda} \equiv \frac{1}{N_{\text {sites }}} \sum_{x} \operatorname{Tr}\left(\lambda \partial_{\mathrm{t}} A_{\mathrm{t}}+\sum_{i=1}^{3} \partial_{i} A_{i}(x)\right)^{2} .
$$

For $0 \leq \lambda \leq 1, \epsilon_{\lambda} \approx 10$ in $\Sigma$ gauge at $\beta=5.7$. Typically, we find for $0 \leq \lambda \leq 1$ that reducing $\epsilon_{\lambda}$ to $\leq 5 \times 10^{-3}$ is sufficient to yield a stable quark propagator signal.

\section{RESULTS}

Our zero momentum $\lambda$ gauge quark propagators may be parametrized as

$$
\begin{array}{cc} 
& <\sum_{\vec{x}} q_{\alpha}^{a}(\vec{x}, t) \bar{q}_{\beta}^{b}(\overrightarrow{0}, 0)>^{\lambda} \\
=\quad & {\left[A^{\lambda}(t) \delta_{\alpha \beta}+B^{\lambda}(t)\left(\gamma_{0}\right)_{\alpha \beta}\right] \delta^{a b}} \\
\underset{t \rightarrow \infty}{\longrightarrow} & {\left[A_{\infty}^{\lambda} \exp ^{-m, t} \delta_{\alpha \beta}\right.} \\
& \left.\quad+B_{\infty}^{\lambda} \exp ^{-m_{v} t}\left(\gamma_{0}\right)_{\alpha \beta}\right] \delta^{a b}
\end{array}
$$

Color indices $a$ and $a$ run from 1 to 3 and $m_{s}$ and $m_{v}$ are masses of the lightest fermions which couple to $q$. After configuration averaging. time reversal invariance - respected by the latticeimplies

$$
\begin{aligned}
& A^{\lambda}(-t)=+A^{\lambda}(t) \\
& B^{\lambda}(-t)=-B^{\lambda}(t) .
\end{aligned}
$$

We have results for the following lattices (configuration size. propagator size. $\beta$. number of configurations used.)

- $\left(6^{3} \times 10.6^{3} \times 17,5.7,32\right) \quad \mathrm{A}$

- $\left(10^{3} \times 10,10^{3} \times 20,5.7,18\right) \quad B$

- $\left(16^{3} \times 24,16^{3} \times 25,5.7,16\right) \quad$ C

- $\left(16^{3} \times 40,16^{3} \times 39.6 .0 .19\right) \quad D$

- $\left(24^{3} \times 40,24^{3} \times 39,6.0 .8\right) \quad$ E

The $6^{3} \times 10$ lattice is used mainly for testing and its data is not reported here. The $16^{3} \times 40$ analysis is not complete. For purposes of orientation. 
we recall that a free quark propagator satisfies the Dirac equation

$$
\left(\gamma_{0} \frac{\partial}{\partial t}-m\right)<\sum_{\vec{x}} q_{\alpha}^{a}(\vec{x}, t) \vec{q}_{\beta}^{b}(\overrightarrow{0}, 0)>^{\text {free }}=0 .
$$

This requires $A=B=$ constant and $m_{\mathbf{s}}=m_{v}$. Figure 1 indicates that our QCD propagators are asymptotically consistent with these expectations for a free quark case. Figure $1 a$ compares

$$
\mathcal{A}(t) \equiv \frac{1}{2}(A(t)+A(-t))
$$

with

$$
B(t) \equiv \frac{1}{2}(B(t)-B(-t))
$$

for the Landau gauge propagator. Although the two quantities are different when $t$ is small. they are the same within errors at sufficiently large $t$. Figure $1 b$ depicts the ratio $\frac{B}{A}$. Errois shown in Figure 1 -as all our other errors-are jackknife errors.

Figure 2 depicts the effective masses $m_{s}$ and $m_{v}$ as a function of time. Like the functions $A$ and $B$. the two masses are the same within errors at large times. Therefore. we do not try to distinguish between $m_{s}$ and $m_{v}$. Table 1 reports a "dynamical" mass value $m_{D}(K)$ which is the asymptotic value of $m_{z}$ for hopping parameter $K$. The scalar signal is quoted because it is the stronger one.

\section{SCALING}

$m_{D}^{\text {critical }}$, the value of $m_{D}(K)$ when $K=K_{e}$, is particularly important. Not only is it a measure of the dynamically generated quark mass when the pion mass vanishes but. being a uniquely defined point, $m_{D}^{\text {critical }}$ permits an unambiguous check on scaling. Unfortunately. lattice propagators cannot be calculated directly at $K_{c}$ and $m_{D}^{\text {critical must be }}$ determined by extrapolation. We make this extrapolation based on two assumptions: the chiral perturbation theory relation $m_{\text {current }} \propto M_{\text {meson }}^{2}$ and the relation $m_{D} \propto$ ( $m_{\text {current }}+$ constant $)$. which is certainly true at large masses and is simplest assumption at small masses. We use for $M_{\text {meson }}(K)$ the lattice mass of the pseudoscalar meson constructed from a degenerate quark-antiquark pair. These assumptions imply

$$
m_{D}(K)=C M_{\text {meson }}^{2}(K)+m_{D}^{\text {critical }} .
$$

As illustrated in Figure 3, this relation is consistent with our data in the low mass regime.

Table 2 has our $C$ and $m_{D}^{\text {critical }}$ values. $m_{D}^{\text {critical }}$ is barely consistent within errors between $\beta=5.7$ and 6.0: whereas the slope $C$ is probably showing scaling violations. Of course, one has no reason to expect good scaling between $\beta=5.7$ and $\beta=6.0$. Note that, since $m_{D}^{\text {critical }}$ and $C$ are dimensionful parameters. different identifications of the $a^{-1}$ values will affect the apparent scaling behavior. Our analysis assumes that $a^{-1}=1.0 \mathrm{GeV}$ for $\beta=5.7$ and $a^{-1}=1.7 \mathrm{GeV}$ for $\beta=6.0$. We believe that these values are uncertain by about $20 \%$. Clearly. tests at weaker coupling are needed.

\section{GAUGE DEPENDENCE}

Gauge dependence of the quark mass defined here is a nontrivial issue. For example, it has been argued that axial gauge masses should differ from other gauges. ${ }^{5}$ However. this does not necessarily mean that quark mass is not gauge invariant within a subclass of gauges, namely, the covariant gauges or our $\lambda$ gauges.

As Table 3 shows, the $m_{D}^{\text {critical signal in }}$ Coulomb gauge is significantiy noisier than in Landau gauge. This is especially true of the $\beta=5.7$ data which is poor enough to inhibit any meaningful comparison. On the other har.d, the $\beta=6.0$ data for the $24^{3} \times 40$ lattice is not bad and $m_{D}^{\text {critical }}$ in Coulomb gauge is consisterit with its Landau gauge value.

We anticipate a better signal in other $\lambda \neq 0$ gauges and we will say more about gauge dependence in the future. ${ }^{6}$

\section{SUMMARY}

Results of our lattice study of the quenched QCD quark propagator in Landau and Coulomb gauges are reported. We find that $m_{D}^{\text {critical }}$. the value of the quark propagator pole in the chiral limit, is about $350 \mathrm{MeV}$. Within the not very stringent limits of our data, $m_{D}^{\text {critical }}$ is barely consistent with scaling and gauge independence. On the other hand, the slope of $m_{D}$ us. $M_{\text {meson }}^{2}$ seems to show significant non-scaling behavior between $\beta=5.7$ and $6.1^{7}$. Clearly, more definite conclusions await 
data at weaker coupling. improvements in statistics. and tests of other $\lambda$ gauges. Such work is in progress. 6

\section{ACKNOWLEDGER.'FNTS}

We are very grateful to Jeff Mandula and Michael Ogilvie for helpful discussions. The computing was done at the National Magnetic Fusion Energy Computing Center (in part under the "Grand Challenge" program) and at the San Diego Supercomputer Center. The research of C.B. was supported in part by the National Science Foundation under Grant No. PHY82-17853. supplemented by funds from NASA.

\section{REFERENCES}

1. J. Mandula. M. Ogilvie, Phys. Lett. B185 (1987) 127

2. See, e.g., E. Fahri and R. Jackiw, eds.. Dynamical Gauge Symmetry Breaking. (World Scientific. Singapore, 1982)

3. See, e.g., A. Carter and H. Pagels. Phys. Rev. Lett. 43, 1845 (1979)

4. H. Pagels, Phys. Rev. D19 (1979) 3080. 1979

5. C. DeTar. J. King. Nucl. Phys. B249 (1985) 644

6. C. Bernard. D. Murphy. A. Soni, K. Yee in progress.

7. We have also looked at $z$ (the value of the residue at the position of the pole). As usual. this is a hard quantity to extract and preliminary data indicated it to be about 2 .

\begin{tabular}{|c|c|c|}
\hline Lattice $(3)$ & $K$ & $m_{D}(K) \mathrm{GeV}$ \\
\hline $10^{3} \times 20(5.7)$ & .094 & $1.83 \pm .09$ \\
& .155 & $.67 \pm .03$ \\
& .160 & $.57 \pm .04$ \\
& .164 & $.48 \pm .04$ \\
& .166 & $.42 \pm .04$ \\
& & \\
$16^{3} \times 25(5.7)$ & .094 & $1.80 \pm .08$ \\
& .155 & $.58 \pm .02$ \\
& .160 & $.49 \pm .02$ \\
& .164 & $.41 \pm .02$ \\
& .166 & $.36 \pm .02$ \\
& .168 & $.33 \pm .02$ \\
& .152 & $.56 \pm .19$ \\
$16^{3} \times 39(6.0)$ & .154 & $.52 \pm .17$ \\
& .155 & $.58 \pm .12$ \\
& .156 & $.51 \pm .23$ \\
& .118 & $1.74 \pm .04$ \\
& $.54 \pm .02$ \\
& .152 & .54 \\
& .155 & $.46 \pm .03$ \\
& $.43 \pm .03$ \\
\hline
\end{tabular}

Table 1: $m_{D}$ is the (dynamical) quark mass measured in Landau gauge. Only statistical errors are shown; $a_{5.7}^{-\frac{1}{1}}=1.0 \mathrm{GeV}, a_{6.0}^{-1}=1.0 \mathrm{GeV}$ are used.

\begin{tabular}{|c|c|c|}
\hline Lattice $(\beta)$ & $m_{D}^{\text {critical }} \mathrm{GeV}$ & $C\left(\mathrm{GeV}^{-1}\right)$ \\
\hline $10^{3} \times 20(5.7)$ & $.35 \pm .05$ & $.43 \pm .05$ \\
$16^{3} \times 25(5.7)$ & $.29 \pm .02$ & $.40 \pm .03$ \\
$16^{3} \times 39(6.0)$ & $.56 \pm .15$ & $-.02 \pm .30$ \\
$24^{3} \times 39(6.0)$ & $.35 \pm .04$ & $.27 \pm .03$ \\
\hline
\end{tabular}

Table 2: Landau gauge results. See equation (13) for notation. See also Table 1.

\section{DISCLAIMER}

This report was prepared as an account of work sponsored by an agency of the United States Government. Neither the United States Government nor any agency thereof, nor any of their employees. makes any warranty, express or implied, or assumes any legal liability or responsibility for the accuracy, completeness. or usefulness of any information, apparatus, product, or process disclosed, or represents that its use would not infringe privately owned rights. Refer. ence herein to any specific commercial product, process, or service by trade name, trademark. manufacturer, or otherwise does not necessari,j constitute or imply its endorsement, recommendation, or favoring by the United States Government or any agency thereof. The vitws and opinions of authors expressed herein do not necessarily state or reflect those of the 


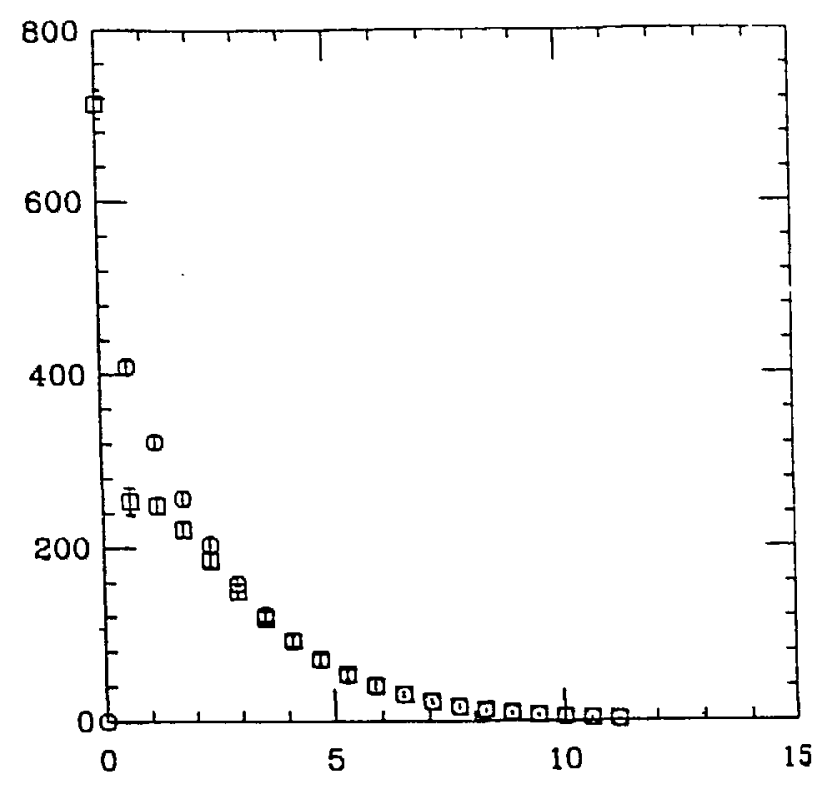

Fig. 1a. The rectangles represents the scalar propagator $A(t)$, as a function of time; the octagon is for the vector propagator, $B(t)$. See equation ( 7 ). Data shown is for $24^{3} \times 40$ lattice, $\beta=6.0, k=.154,8$ configurations.

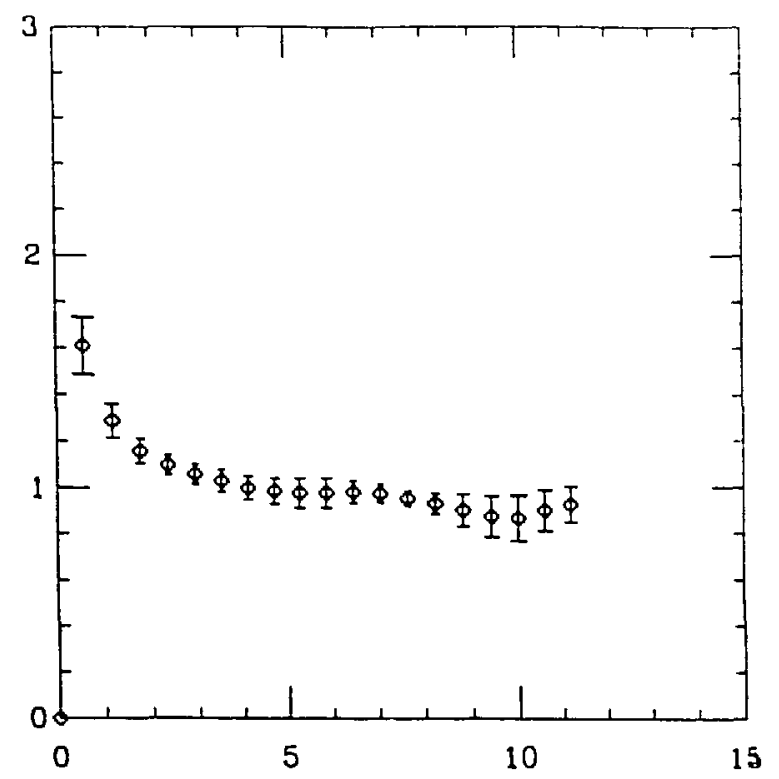

Fig. 1b. The Ratio $A(t) / B(t)$ as a function of time. See caption to Fig. 1a.

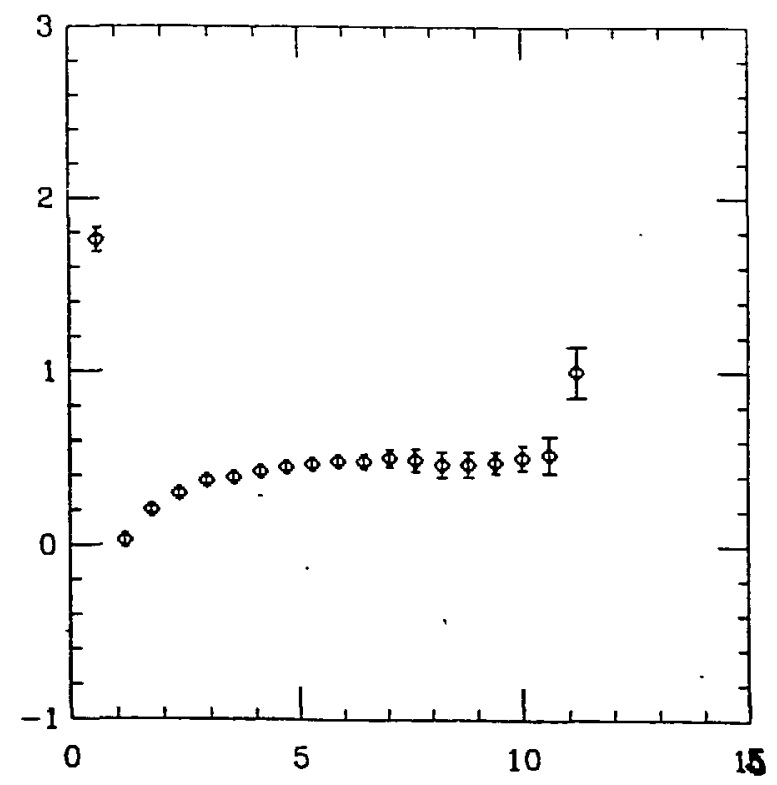

Fig. 2. The quark (dynamical) mass, as a function of time. Same data as in Fig. 1. 


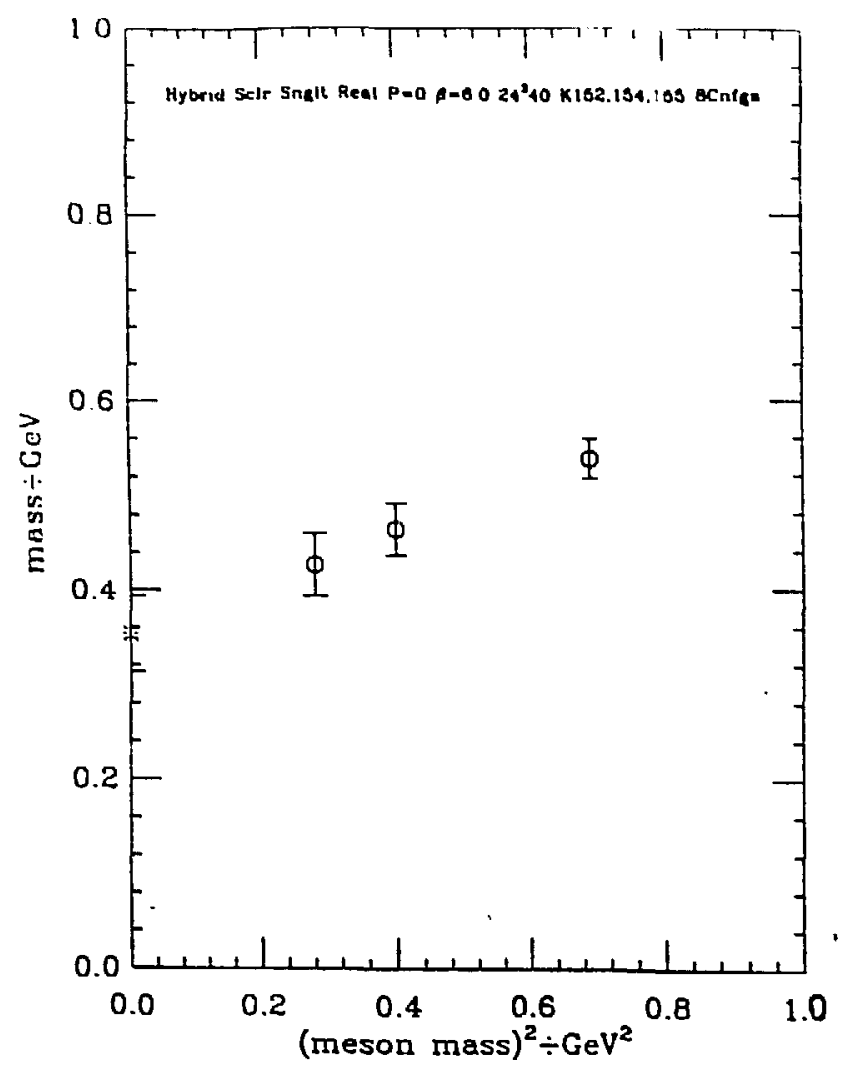

Fig. 3a. $m_{D}(k)$ versus $m_{\text {meson }}^{2}(k)$ [see Eq. (13)] for $24^{3} \times 40, \beta=6.0$ lattice.

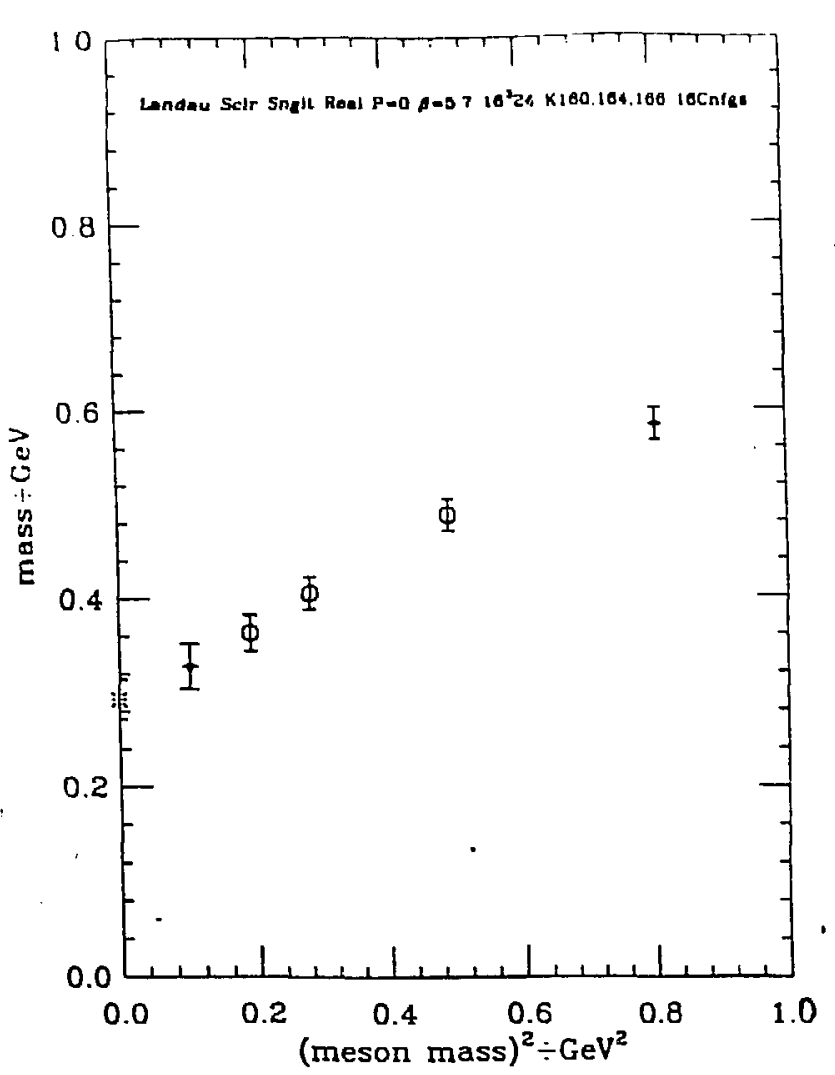

Fig. 3b. $m_{D}(k)$ versus $m_{\text {meson }}^{2}(k)$ for $16^{3} \times$ $24, \beta=5.7$ lattice. 